\title{
Protective Effect of Pulp Oil Extracted from Canarium odontophyllum Miq. Fruit on Blood Lipids, Lipid Peroxidation, and Antioxidant Status in Healthy Rabbits
}

\author{
Faridah Hanim Shakirin, ${ }^{1,2}$ Azrina Azlan,, ${ }^{2,3}$ Amin Ismail, ${ }^{2,3}$ \\ Zulkhairi Amom, ${ }^{4}$ and Lau Cheng Yuon ${ }^{5}$ \\ ${ }^{1}$ Department of Biochemistry, Faculty of Biotechnology and Biomolecular Sciences, Universiti Putra Malaysia, \\ 43400 Serdang, Selangor, Malaysia \\ ${ }^{2}$ Department of Nutrition and Dietetics, Faculty of Medicine and Health Sciences, Universiti Putra Malaysia, \\ 43400 Serdang, Selangor, Malaysia \\ ${ }^{3}$ Laboratory of Halal Science Research, Halal Products Research Institute, Universiti Putra Malaysia, \\ 43400 Serdang, Selangor, Malaysia \\ ${ }^{4}$ Department of Basic Sciences, Faculty of Health Sciences, Universiti Teknologi MARA, 42300 Puncak Alam, \\ Selangor Darul Ehsan, Malaysia \\ ${ }^{5}$ Department of Agriculture, Agriculture Research Centre, Semongok, 93720 Kuching, Sarawak, Malaysia
}

Correspondence should be addressed to Azrina Azlan, azrina@medic.upm.edu.my

Received 1 February 2012; Accepted 14 March 2012

Academic Editor: Oren Tirosh

Copyright ( 2012 Faridah Hanim Shakirin et al. This is an open access article distributed under the Creative Commons Attribution License, which permits unrestricted use, distribution, and reproduction in any medium, provided the original work is properly cited.

\begin{abstract}
The aim of this paper was to compare the effects of pulp and kernel oils of Canarium odontophyllum Miq. (CO) on lipid profile, lipid peroxidation, and oxidative stress of healthy rabbits. The oils are rich in SFAs and MUFAs (mainly palmitic and oleic acids). The pulp oil is rich in polyphenols. Male New Zealand white (NZW) rabbits were fed for 4 weeks on a normal diet containing pulp (NP) or kernel oil (NK) of CO while corn oil was used as control (NC). Total cholesterol (TC), HDL-C, LDL-c and triglycerides (TG) levels were measured in this paper. Antioxidant enzymes (superoxide dismutase and glutathione peroxidise), thiobarbiturate reactive substances (TBARSs), and plasma total antioxidant status (TAS) were also evaluated. Supplementation of CO pulp oil resulted in favorable changes in blood lipid and lipid peroxidation (increased HDL-C, reduced LDL-C, TG, TBARS levels) with enhancement of SOD, GPx, and plasma TAS levels. Meanwhile, supplementation of kernel oil caused lowering of plasma TC and LDL-C as well as enhancement of SOD and TAS levels. These changes showed that oils of CO could be beneficial in improving lipid profile and antioxidant status as when using part of normal diet. The oils can be used as alternative to present vegetable oil.
\end{abstract}

\section{Introduction}

Canarium odontophyllum Miq. (CO) or Dabai is an indigenous fruit of Sarawak, Malaysia. The fruit is locally consumed by communities in Sarawak and categorized as underutilized. The fruits are oblong in shape measuring $3-4 \mathrm{~cm}$ in length and weighing 10-13 g [1]. The fruit is highly nutritious and rich in minerals, proteins, carbohydrate, and fat. Fat is the major macronutrient in CO being $21-25 \%$ in pulp and $70 \%$ in kernel. Previous study by Azrina et al. [2] revealed that $44.4 \%$ and $60.8 \%$ of saturated fatty acids (SFAs) were found in Dabai pulp and kernel oils, respectively. These oils are rich in palmitic (16:0) and oleic (18:0) acids with fatty acid composition of the pulp oil similar to palm oil. $\mathrm{CO}$ fruit is also high in phenolic compounds and vitamin E such as $\gamma$-tocopherol $[2,3]$. The high phenolic content of the pulp and kernel has been associated to substantial antioxidant activities as studied by Shakirin et al. and Chew et al. [3, 4]. Chew et al. [4] showed that CO fruit pulp exhibited the most significant antioxidant activities, using 
Trolox equivalent antioxidant capacity (TEAC) and ferric reducing ability (FRAP) assays, while Shakirin et al. [3] studied CO fruit using 1,1-diphenyl-2-picrylhydrazyl radical $(\mathrm{DPPH}), \beta$-carotene bleaching and ferric-reducing ability (FRAP) assays. Both of these studies indicated that the antioxidant activities of the fruit were highly correlated with the total phenolic and flavonoid contents of the fruit. To date, only fatty acid composition of pulp and kernel oils of CO fruit was studied. Therefore, this paper aims to explore fatty acids and total phenolic contents of the oils as well as to assess their protective effects against CHD indicators in healthy rabbits.

\section{Materials and Methods}

2.1. Plant Material. Fresh fruits of $\mathrm{CO}$ were obtained from Agriculture Research Centre, Semongok, Sarawak, Malaysia. The herbarium voucher specimens (S64872) were identified and deposited in the Agriculture Research Centre, Sarawak, Malaysia. The fruits were packed in an ice box $\left(-4^{\circ} \mathrm{C}\right)$ and transported on the same day to Peninsular Malaysia by air plane and delivered to Universiti Putra Malaysia. Upon arrival, fruits without any physical damage were selected and stored at $-20^{\circ} \mathrm{C}$ (Haier Co., Ltd., Hong Kong, China) prior to analysis.

2.2. Sample Preparation. Firstly, the separated fruits were thawed for several minutes at room temperature. Then, the fruit pulp was separated manually, freeze dried (35 XL, Virtis Co. Inc., New York), and ground. The pulp powder was used to extract oil using chloroform-methanol $(2: 1 \mathrm{v} / \mathrm{v})$ at a ratio of $1: 5(\mathrm{w} / \mathrm{v})$. The oil obtained was used for fatty acid composition determination and supplementation to animals. Kernel powder of the fruit was obtained by cracking the seeds, pooled, freeze dried, and prepared the same way as pulp.

2.3. Analysis of Fatty Acid Composition. The fatty acid composition of pulp and kernel oils was determined by gas chromatography (Hewlett-Packard, HP1100, USA Agilent Technology). Fatty acid methyl esters (FAMEs) were prepared by method as described by Frank et al. [5]. Briefly, $100 \mathrm{mg}$ of sample was weighed in a $20 \mathrm{~mL}$ test tube (with screw cap). Then, the sample was dissolved in $10 \mathrm{~mL}$ of hexane. Next, $100 \mu \mathrm{L}$ of $2 \mathrm{~N}$ potassium hydroxide in methanol was added. After vortex (BOECO, Germany) for 30 seconds, the mixture was centrifuged. Next, $2 \mathrm{~mL}$ of the clear supernatant was transferred to a sample vial and analyzed using gas chromatography.

2.4. Gas Chromatography (GC) Condition. The fatty acid composition of the oil samples was analyzed using an Agilent 6890 GC (USA Agilent Technology) equipped with split splitless injector, detector, and Hewlett-Packard EL-980 flame ionization detection (FID) system to separate and quantify each FAME component. FAMEs were separated using DB-23 column $(60 \mathrm{~m} \times 0.25 \mathrm{~mm}$ I.D., $0.15 \mu \mathrm{m}$ polyethylene glycol film). Chromatography data were recorded and integrated using Chemstations software (Version 6.0). The oven temperature was held at $50^{\circ} \mathrm{C}$ for $1 \mathrm{~min}$, then increased to $175^{\circ} \mathrm{C}$ at $4^{\circ} \mathrm{C} / \mathrm{min}$, and lastly increased to $230^{\circ} \mathrm{C}$, holding for $5 \mathrm{~min}$ at $230^{\circ} \mathrm{C}$. The temperatures for the injector and detector were set at $250^{\circ} \mathrm{C}$ and $280^{\circ} \mathrm{C}$, respectively. One microliter of the sample volume was injected with a split ratio of $1: 50$ at a column temperature $110^{\circ} \mathrm{C}$. Carrier gases that were used in the system were helium gas, $1.0 \mathrm{~mL} / \mathrm{min}$ controlled at $103.4 \mathrm{kPa}$; the hydrogen and air used for FID was held at $275.6 \mathrm{kPa}$.

\subsection{Determination of Total Phenolic Content in Pulp and Kernel Oils}

2.5.1. Preparation of Polar Fraction. Polar fraction was prepared by method developed by Gutfinger [6]. First, 10 grams of oil was mixed with $50 \mathrm{~mL}$ hexane. Then, the solution were extracted successfully with $20 \mathrm{~mL} 60 \%$ methanol for three times. The extracts were combined and concentrated under reduced pressure using rotary evaporator (Buchi Rotovapor R-200, Berlin, Germany) at $36^{\circ} \mathrm{C}$. The concentrated extract was dissolved in $5 \mathrm{~mL}$ methanol and considered as polar fraction. The polar fraction was used in the determination of total phenolic content.

2.6. Determination of Total Phenolic Content. Total phenolic content of the polar fraction was determined according to method of Singleton and Rossi [7] with some modifications as described by Velioglu et al. [8]. One hundred microliter of polar extract was mixed with $0.75 \mathrm{~mL}$ of Folin Ciocalteu reagent (10-fold dilution with distilled water) and allowed to stand at $22^{\circ} \mathrm{C}$ for five minutes. Then $0.75 \mathrm{~mL}$ of sodium carbonate $(60 \mathrm{~g} / \mathrm{L})$ solution was added to the mixture. After $90 \mathrm{~min}$ at $22^{\circ} \mathrm{C}$, absorbance was measured at $725 \mathrm{~nm}$ using a UV-visible Shimadzu spectrophotometer (UV 1601, Shimadzu Corporation, Victoria, Australia). Gallic acid $(0.02-0.1 \mathrm{mg} / \mathrm{mL})$ was used as standard. The total phenolic content was calculated for each sample. Samples were run in triplicates, and the result was expressed in mg GAE per $\mathrm{kg}$ of sample.

2.7. Animals and Diets. Fourteen male New Zealand white (NZW) rabbits weighing 1.5-1.7 kg at age of 8-10 weeks were purchased from East Asia Company, Malaysia. Rabbits were housed individually in wire-bottom cages and maintained on 12-hour light-dark cycle in a climate-controlled room $\left(20^{\circ} \mathrm{C}-24^{\circ} \mathrm{C}, 40 \%-45 \%\right.$ relative humidity). Food and water were made available ad libitum throughout the study. All procedures were reviewed and approved by the Animal Care and Use Committee of the Faculty of Medicine and Health Sciences, UPM (approval number: UPM/FPSK/PADS/BR$\mathrm{UUH} / 00238$ ).

The rabbits were acclimatized for two weeks before treatment. The animals were randomly divided into two groups (seven animals for each group). They were normal group (NC, receive normal diet) and supplemented group (NP or NK). The supplemented group was started by receiving NP diet (normal diet daily $+2 \%$ pulp oil) followed 
by NK diet (normal diet $+2 \%$ kernel oil) (Figure 1). Supplementation of oil was by force feeding. All diets (NC, $\mathrm{NP}$ and NK) were provided for 4 weeks before termination. There was a two-week wash-out period between NP and NK supplementation. Ingredients used in the preparation of purified diets are as shown in Table 1. The four-week study period followed a previous study by Basu et al. [9] who showed that supplementation of vegetable oil/fat (seabuckthorn oil) for 18 days improved the lipid profile of healthy rabbits. This study, however, used longer duration of 4 weeks to observe for more pronounced effect of the pulp and kernel oils of CO.

2.8. Experimental Design and Procedures. Body weight and food intake were measured before and after the experiment. The rabbits were fasted for 12 hours before blood sampling. Blood was drawn from marginal ear of rabbits at baseline and week 4. Blood was collected into different test tubes. Blood for plasma lipid profiles and malondialdehyde (MDA) was collected in tubes containing ethylenediaminetetraacetic acid (EDTA) while blood for determining superoxide dismutase (SOD), glutathione peroxidase (GPx), and total antioxidant status (TAS) was collected in tubes containing lithium heparin. Both EDTA and lithium heparin are anticoagulant. The collected blood was centrifuged at $3000 \mathrm{rpm}$ for $10 \mathrm{~min}$ (Universal 32, Hettich Zentrifugen, Germany) to separate the plasma. For SOD and GPx test, fresh blood was required to determine the enzyme activity.

2.9. Biochemical Measurement. Total cholesterol (TC), lowdensity lipoprotein cholesterol (LDL-C), and high-density lipoprotein cholesterol (HDL-C) were assessed using Roche Diagnostic (Germany), while total antioxidant status (TAS), superoxide dismutase (SOD), and glutathione peroxidase $(\mathrm{GPx})$ kits were assessed using Randox Laboratory Ltd (United Kingdom). Plasma MDA levels were determined according to method described by Beuge and Aust [10].

2.10. Statistical Analysis. The data were presented as group means \pm SEM. SPSS (Statistical Package Social Sciences) version 16.0 (SPSS Corporation, Chicago, IL) for Windows was used to analyze the data. Differences between group means were determined using one-way Analysis of Variance (ANOVA). The Tukey post hoc test was used for multiple group comparison. The significance value was set at $P<0.05$.

\section{Results}

Table 2 shows fatty acid composition of pulp and kernel oils of CO. Both oils are rich in palmitic acid (16:0) and oleic acid (18:1). For pulp oil, equal amount of saturated fatty acids (SFAs) and monounsaturated fatty acids (MUFAs) was found. Meanwhile, the level of SFAs in kernel oil was much higher compared to MUFAs. Table 3 shows the total phenolic content (TPC) of pulp and kernel oils extracted from CO fruit. Higher TPC was found in pulp oil compared to kernel oil.
Between the two groups, food intake in animals fed with pulp oil (NP) was higher than animals fed on kernel oil (NK) (Figure 2). In contrast, after 4 weeks, animals on pulp oil had lower body weight while animals on kernel oil had similar body weight as normal group (NC) (Figure 2).

Feeding pulp oil to healthy rabbits resulted in significant increment of TC level compared to rabbits fed on normal diet. Meanwhile, feeding kernel oil resulted in significant decrease of TC level after 4 weeks of treatment (Figure 3(a)). Alternatively, feeding pulp oil resulted in increased HDL-c level and significant decrease of LDL-c $(P<0.05)$. Only slight elevation of HDL-c and moderate reduction of LDL-c levels occurred when rabbits were fed with kernel oil (Figures 3(b) and $3(\mathrm{c}))$. The feeding of pulp oil (NP) also resulted in a significant reduction of $\mathrm{TG}$, while only slight increased of TG level in rabbits fed with kernel oil (NK) was observed (Figure 3(d)).

Supplementation of pulp oil resulted in significant reduction $(P<0.05)$ of plasma MDA level compared to normal group. The MDA level, however, was significantly increase $(P<0.05)$ by 2 folds in rabbits fed with kernel oil compared to normal group (Figure 4(a)). Supplementation with kernel oil resulted in significant increase $(P<0.05)$ in SOD activity instead and no significant change of GPx activity compared to normal group. On contrary, supplementation with pulp oil resulted in significant increase $(P<0.05)$ of SOD and GPx activities as compared to rabbits fed on normal diet. The TAS levels of groups supplemented with kernel and pulp oils, respectively, were increased significantly $(P<0.05)$ compared to normal group (Figures $4(\mathrm{~b})$ and $4(\mathrm{c})$ ).

\section{Discussion}

4.1. Fatty Acid Composition. Fatty acid composition of pulp oil of CO in this study (Table 2) was comparable to report by Nurnadia [11] with slightly higher PUFA found in this study (14.05\% versus $12.76 \%)$. Another study by Ibrahim et al. [12] using Canarium odontophyllum from Brunei found comparable SFAs (42.2\%), lower MUFAs (36.4\%), and PUFAs (13.5\%) contents compared to this study. In this study, palmitic acid (16:0), oleic acid (18:1), and linoleic acid (18:2 n6 cis) were the main fatty acids present in the pulp oil of CO. Low levels of myristic acid (14:0) and arachidic acid (20:0) were found in the pulp. Sample of this study also had lower palmitic acid compared to C. schweinfurthii pulp oil (40.31\% versus $60.88 \%$ ) [13]. Total MUFAs of the CO pulp oil reported in this study also were much lower compared to high-fat fruits such as olive (56-86\%) and avocado (65-68\%) $[14,15]$. Meanwhile, PUFA of the CO pulp oil (14\%) was comparable to avocado oil (12-14\%) but lower than olive oil $(3.5-21.5 \%)[14,15]$.

Generally, fatty acid composition (FAC) of the pulp oil is similar to palm oil with equal amount of SFAs and UFAs characterized mainly by palmitic (16:0) and oleic acids (18:1). Palm oil is commercially used as vegetable/cooking oil due to its fatty acid stability that is distinct from other vegetable oils. Palm oil has been widely used in broad food production and preparation as it requires little or 
TABLE 1: Ingredients of experimental diets $(\mathrm{g} / \mathrm{kg})$.

\begin{tabular}{|c|c|c|c|}
\hline \multirow{2}{*}{ Ingredients } & \multicolumn{3}{|c|}{ Ingredients in experimental groups $(\mathrm{g} / \mathrm{kg})$} \\
\hline & $\mathrm{NC}$ & NP & NK \\
\hline Soybean meal & 150 & 150 & 150 \\
\hline Corn & 300 & 300 & 300 \\
\hline Palm kernel & 360 & 360 & 360 \\
\hline Starch & 100 & 100 & 100 \\
\hline Molasses & 20 & 20 & 20 \\
\hline Corn oil & 20 & - & - \\
\hline Vitamin mixture $^{\mathrm{a}}$ & 3 & 3 & 3 \\
\hline Mineral mixture $^{b}$ & 35 & 35 & 35 \\
\hline DL-methionine & 2 & 2 & 2 \\
\hline $\mathrm{CaCO}_{3}$ & 5 & 5 & 5 \\
\hline $\mathrm{CaHPO}_{4}$ & 5 & 5 & 5 \\
\hline Pulp oil of CO & - & 20 & - \\
\hline Kernel oil of CO & - & - & 20 \\
\hline Total & 1000 & 1000 & 1000 \\
\hline
\end{tabular}

NC (negative control): normal diet,

$\mathrm{NP}$ : normal diet $+2 \%$ pulp oil extracted from CO fruit,

NK: normal diet $+2 \%$ kernel oil extracted from $\mathrm{CO}$ fruit,

${ }^{a}$ Vitamin mixture: vitamine A: 50000 i.u, vitamine D3: 8000 i.u, vitamine E: 8 mg,

b Mineral mixture: manganese: $320 \mathrm{mg}$, zinc: $200 \mathrm{mg}$, magnesium $1400 \mathrm{mg}$,

ferrous: $300 \mathrm{mg}$, opper: $50 \mathrm{mg}$, cobalt: $10 \mathrm{mg}$, iodate: $20 \mathrm{mg}$,

phosphorus: $10000 \mathrm{mg}$, salt: $5500 \mathrm{mg}$ and calcium: $1300 \mathrm{mg}$.

TABLe 2: Fatty acid composition of pulp and kernel oils of CO.

\begin{tabular}{lcc}
\hline Fatty acids & Pulp oil & Percentage $(\%)$ \\
\hline Saturated fatty acid & $43.42 \pm 0.05$ & $56.2 \pm 0.04$ \\
$14: 0$ & $0.31 \pm 0.04$ & $0.1 \pm 0.01$ \\
$16: 0$ & $40.31 \pm 0.01$ & $50.22 \pm 0.74$ \\
$18: 0$ & $2.19 \pm 1.04$ & $5.88 \pm 0.76$ \\
$20: 0$ & $0.61 \pm 0.07$ & ND \\
Monounsaturated fatty acid & $42.53 \pm 0.06$ & $39.84 \pm 0.04$ \\
$16: 01$ & $0.63 \pm 0.08$ & $0.28 \pm 0.05$ \\
$18: 01$ & $41.9 \pm 0.02$ & $39.56 \pm 0.48$ \\
Polyunsaturated fatty acid & $14.05 \pm 0.09$ & $3.96 \pm 0.04$ \\
$18: 2$ n6 cis & $14.05 \pm 1.96$ & $3.73 \pm 0.41$ \\
$18: 3$ n3 & $\mathrm{ND}$ & $0.23 \pm 0.04$ \\
\hline
\end{tabular}

ND is not detected.

Data are expressed as mean \pm standard deviation $(n=3)$.

TABle 3: Total phenolic content of oils extracted from CO fruit.

\begin{tabular}{lc}
\hline Sample & Total phenolic content $(\mathrm{mg} \mathrm{GAE} / \mathrm{kg}$ oil $)$ \\
\hline Pulp oil & $202.70 \pm 6.65^{\mathrm{b}}$ \\
Kernel oil & $39.40 \pm 5.82^{\mathrm{a}}$ \\
\hline
\end{tabular}

Data are expressed as mean \pm standard deviation $(n=3)$ whose different letters are significantly different at $P<0.05$.

no hydrogenation that can prolong the shelf life of food products [16]. Thus, the studied pulp oil of CO is potentially used as new vegetable/cooking oil and food applications in future.

Kernel oil of CO was slightly higher in SFAs than MUFAs and PUFAs, similar to observation by Nurnadia [11]. Ibrahim et al. [12], however, reported lower SFAs $(52.7 \%)$ and MUFAs (36.1\%) but higher PUFAs (4.3\%) in C. odontophyllum from Brunei. In this study, palmitic and oleic acids accounted for more than $80 \%$ of the total fatty acids. Palmitic acid, oleic acid, and linoleic acid were the major SFAs, MUFAs, and PUFAs, respectively. The palmitic and oleic acids of the studied kernel oil were higher compared to previous study [12]. The fatty acid composition of CO kernel 


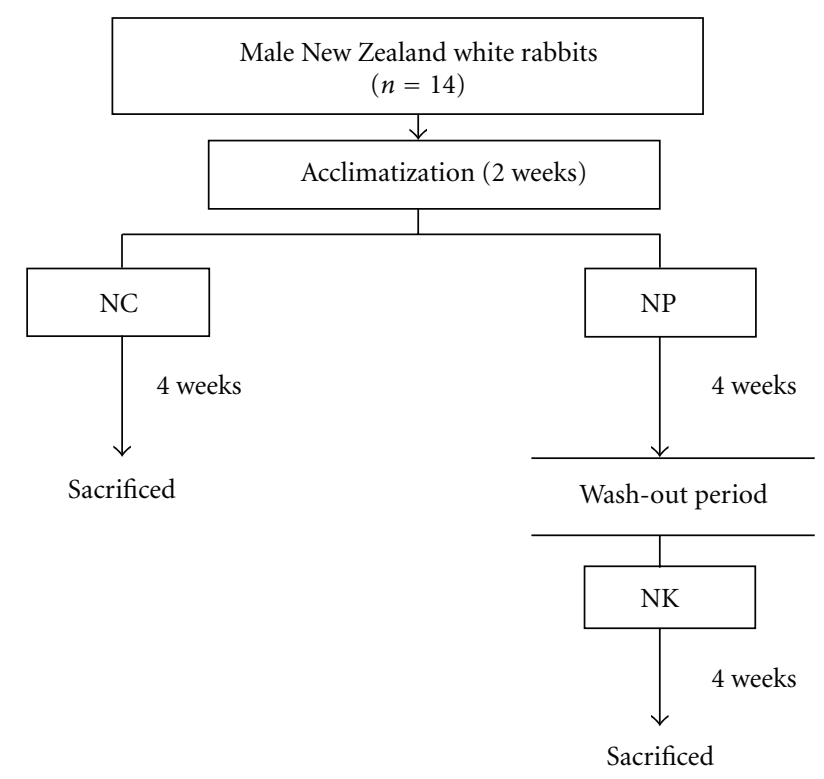

FIgURE 1: Experimental design of the study on the lipid lowering effect of pulp and kernel oils of CO. NC: normal diet group, NP: normal diet + pulp oil of CO, NK: normal diet + kernel oil of CO.

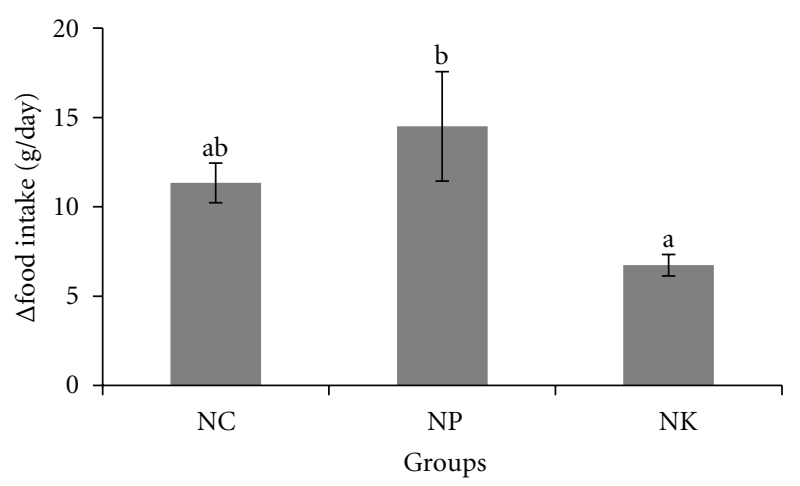

(a)

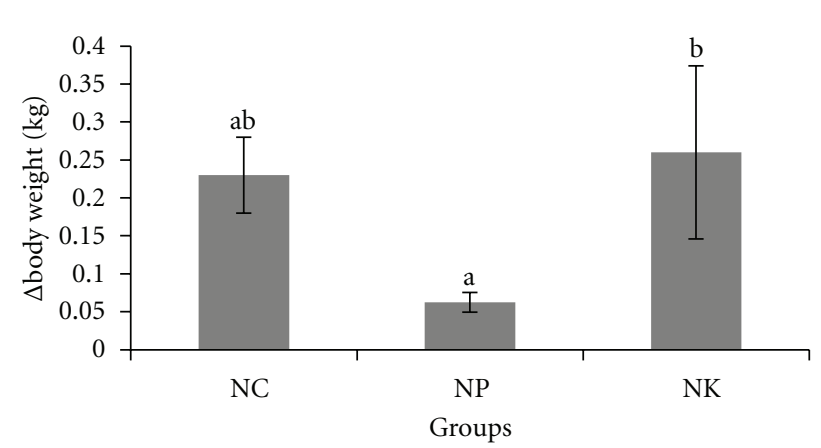

(b)

FIGURE 2: Changes $(\Delta)$ in (a) food intake (b) body weight in rabbits. NC: negative control; NP: $2 \%$ of pulp oil + normal diet; NK: $2 \%$ kernel oil + normal diet. Values are expressed as means \pm S.E.M. Differences between groups were assessed using analysis of variance. Values with different alphabet are significantly different $(P<0.05)$ between groups. $\Delta$ means changes from week 0 to week 4 .

oil was similar to C. ovatum as palmitic and oleic acids were present at $44.4-59.6 \%$ and $32.6-38.2 \%$, respectively [17]. However, higher SFAs content was detected in the studied kernel oil compared to C. album L. [18]. Although kernel oil can be characterized as saturated oil, the oil however was less saturated compared to coconut oil (56\% versus $70 \%$ ). Moreover, CO kernel oil contained less harmful fatty acids (lauric and myristic acids) compared to coconut oil.

4.2. Total Phenolic Content in Oils. Polyphenols are one of the main groups of phenolic compounds acting as primary antioxidants to inhibit oxidation of the oil. They mainly act as chain breaking antioxidant by donating hydrogen to alkylperoxyl radicals $\left(\mathrm{ROO}^{\bullet}\right)$ formed during the initiation step of lipid peroxidation [19]. As CO is categorized as highfat fruit, therefore, it can easily deteriorate. The presence of polyphenols in the fruit is beneficial to reduce oxidation, thus preventing deterioration.

The higher total phenolic content (TPC) in the pulp oil (Table 3) could be due to major contribution of phenolic compounds from the skin. Darker color of the pulp oil was due to the presence of skin pigment that contributed to high content of polyphenols. The TPC in pulp oil was comparable to level in virgin olive oil as determined by Pellegrini et al. [20] where TPC of virgin olive oil (VOO) was in the range of 73-265 mg GAE/kg oil. VOO has been reported to be protective against heart disease, ulcer, and cancer [21]. Consequently, the studied pulp oil could also offer some beneficial effects as VOO following the presence of natural antioxidants that could act as buffer against free radicals [22]. Lower phenolic content in the kernel oil is in agreement with Tuberoso et al. [23] who reported that most of the phenolic 


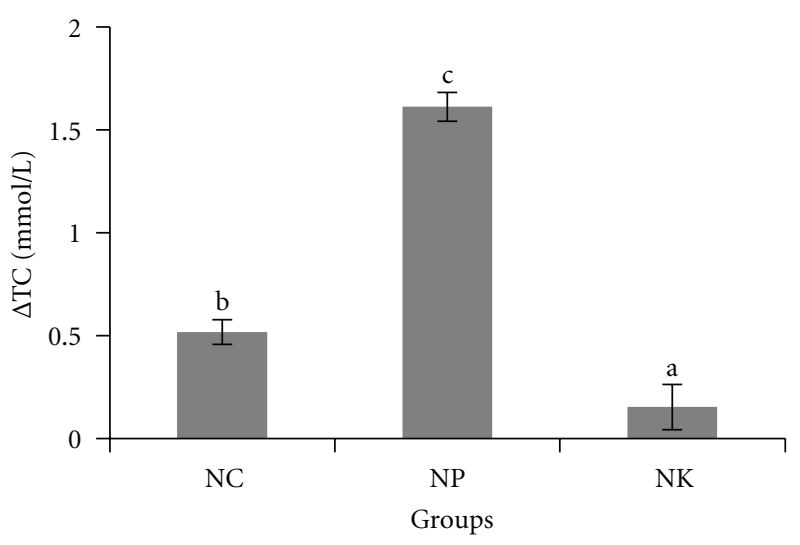

(a)

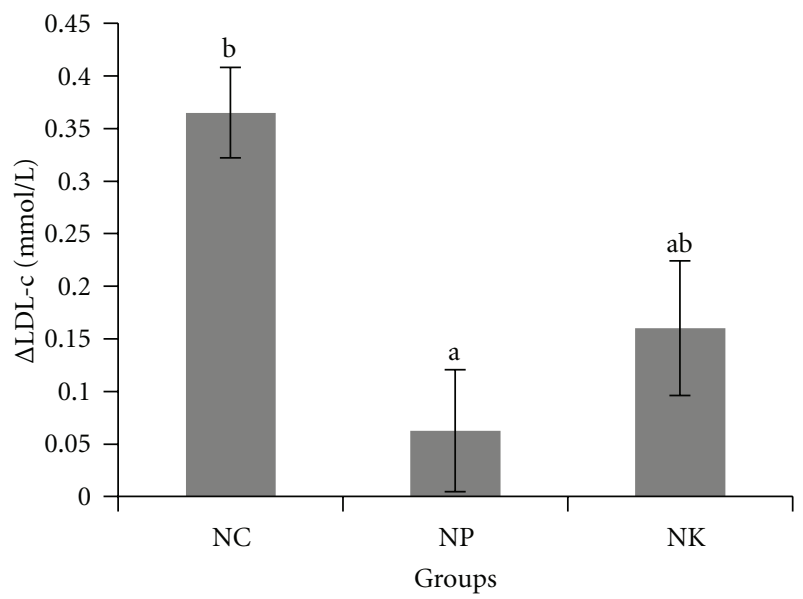

(c)

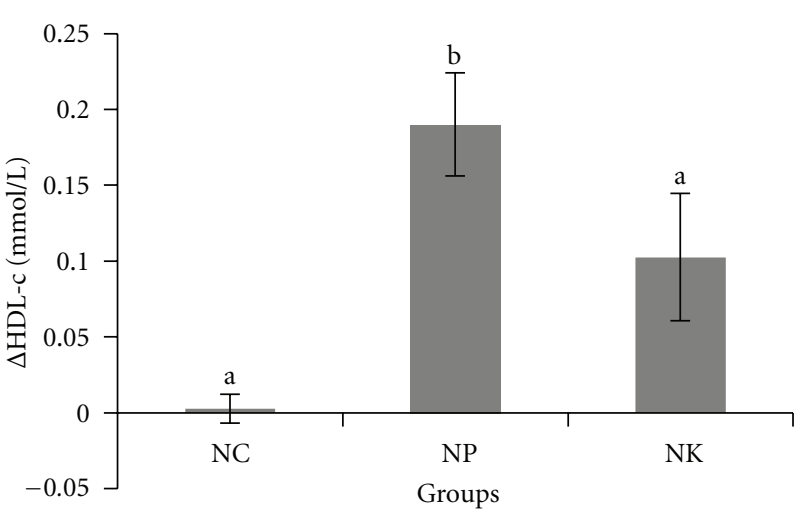

(b)

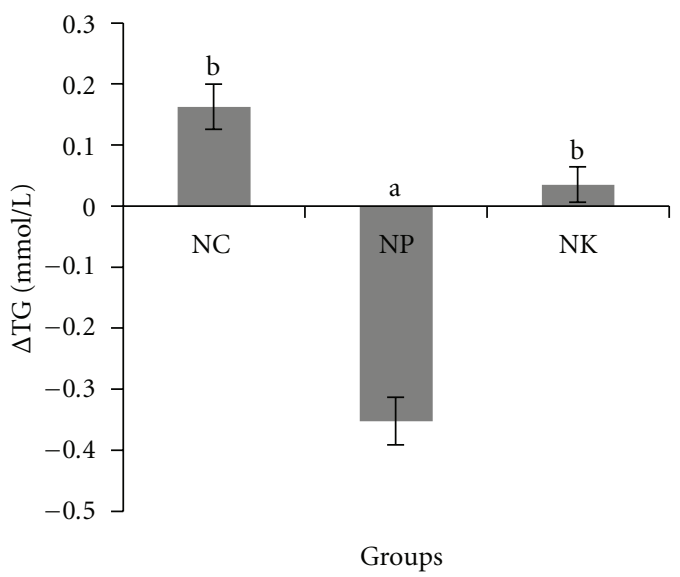

(d)

FIgURE 3: Changes $(\Delta)$ in plasma (a) total cholesterol-TC (b) HDL-c (c) LDL-c (d) TG in rabbits. NC: negative control; NP: $2 \%$ of pulp oil + normal diet; NK: $2 \%$ kernel oil + normal diet. Values are expressed as means \pm S.E.M. Differences between groups were assessed using analysis of variance. Values with different alphabet are significantly different $(P<0.05)$ between groups. $\Delta$ means changes from week 0 to week 4.

compounds are not present in the kernel of fruit such as observed in olive and grape.

4.3. Food Intake and Body Weight. The significantly higher food intake during pulp oil supplementation (Figure 2(a)) observed in this study suggests that the pulp oil may induce satiety compared to kernel oil supplementation. In contrast, supplementation of pulp oil resulted in lower body weight changes although the food intake was higher (Figure 2(a)). This could be attributed to the different types of fat received during the experimental period. The kernel oil contained more SFAs compared to the pulp oil. The consumption of saturated fat may increase body weight as previous studies have demonstrated a linear correlation between saturation of fat and increase in body weight [24, 25]. Tzang et al. [26] also showed that consumption of saturated fat from coconut oil and butter could increase body weight in animals as compared to unsaturated fat from flaxseed.
4.4. Effect of Pulp and Kernel Oils Supplementation on Plasma Lipid Profile. Dietary fats play a considerable role in modulating plasma cholesterol concentration and determining the risk of CHD. The type and proportion of fatty acids present in a diet have particular effect on lipoprotein profiles $[27,28]$. The fatty acid composition of dietary fats constitutes a good indicator to define nutritional value as SFAs are always correlated to increased risk in CHD while MUFAs and PUFAs are correlated with decreased risk of CHD. Studies conducted in humans and animals which discovered the effect of vegetable oils on the plasma lipoprotein in relation to CVD are receiving considerable attention $[29,30]$. In this study, administration of CO pulp oil in healthy rabbits had increased the plasma TC (Figure 3(a)). However, it cannot be concluded that the pulp oil promotes CVD risks as plasma HDL-C was also significantly increased compared to plasma LDL-C (Figures 3(b) and 3(c)). A similar observation has been made by A. Ajayi and D. Ajayi [31] using rats, where TC and HDL-C levels were significantly elevated in rats fed 


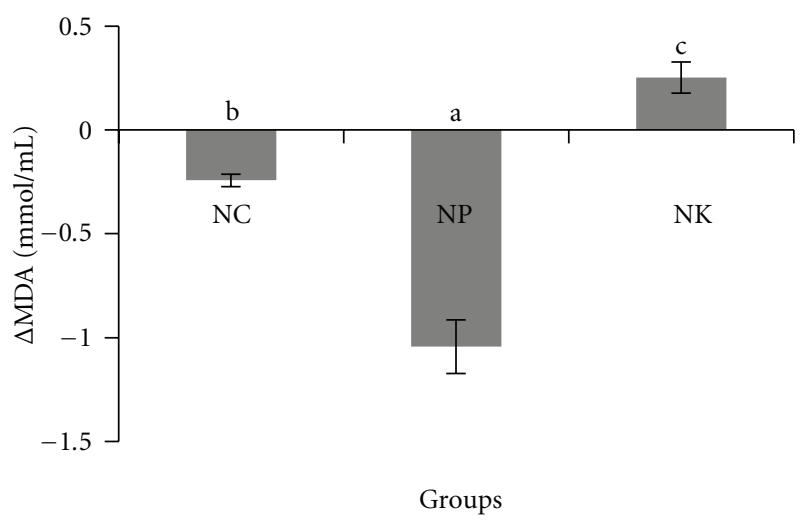

(a)

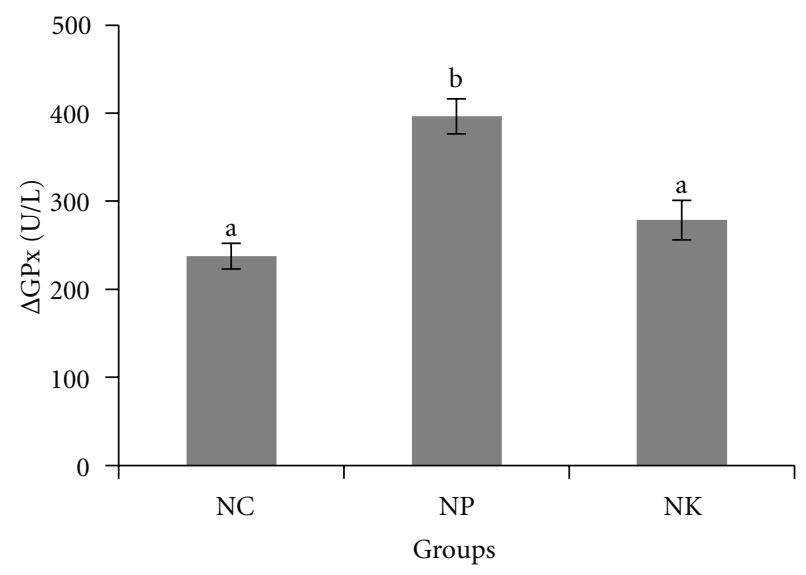

(c)

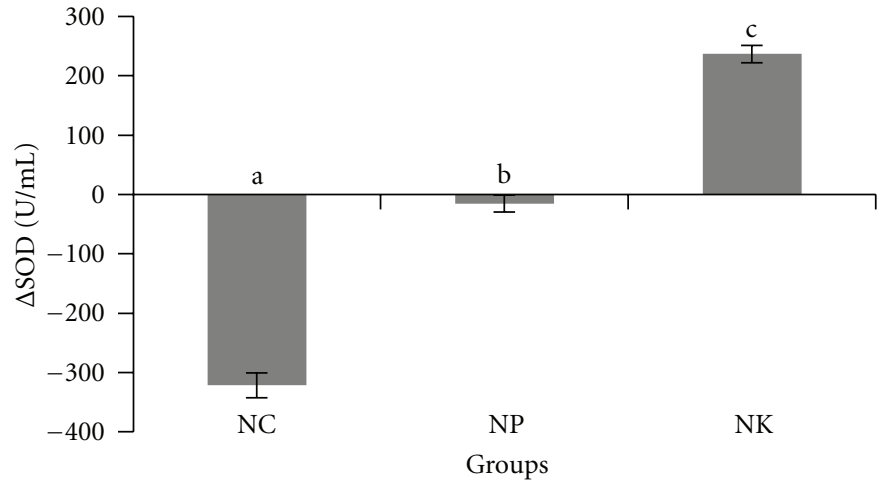

(b)

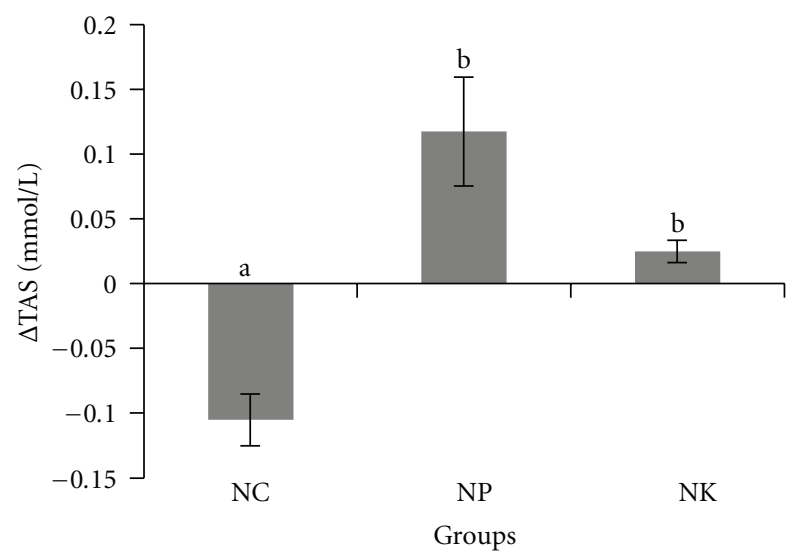

(d)

Figure 4: Changes $(\Delta)$ in (a) plasma MDA level (b) SOD activity (c) GPx activity (d) TAS in rabbits. NC: negative control; NP: 2\% of pulp oil + normal diet; NK: $2 \%$ kernel oil + normal diet. Values are expressed as means \pm S.E.M. Values with different alphabet are significantly different $(P<0.05)$ between groups. $\Delta$ means changes from week 0 to week 4 .

with Origi oil for 6 weeks. There was an inverse relationship between HDL-C and cardiovascular diseases $[9,32]$.

The elevation of LDL-C level may induce formation of oxidized LDL, which plays a key role in inflammation, with lipid accumulation in arterial walls leading to endothelial dysfunction [33]. Plasma LDL-C was lower in both groups supplemented with pulp oil (NP) and kernel oil (NK), respectively, compared to control group. However, the reduction was only significant in the NP group.

There are several mechanisms that could be suggested in relation to the hypocholesterolemic action of pulp and kernel oil. In the animal study, the pulp oil is rich in phenolic compounds especially from skin of $\mathrm{CO}$, attenuating its antioxidative effect and favorable blood lipids [34]. The higher improvement of lipid profile by supplementation of pulp oil compared to kernel oil also was due to the higher content of total phenolics (Table 3). Higher phenolic content contributes to higher antioxidant activities, which protects cell from oxidative damage, DNA impairment, cellular protein, and membrane degeneration [35]. This antioxidant activity could reduce reactive oxygen species (ROS) levels as indicated by reduction in the plasma MDA level. The cholesterol-lowering properties of the pulp oil also could be due to other plant phytochemicals such as sterols. These substances (not measured in this study) are commonly found in oils of plant origin such as soy, corn, peanut, and other plant oils [36]. These phytosterols have shown to be effective in reducing plasma cholesterol. The LDL-C reduction by the pulp oil could be partly due to these compounds.

Different fatty acids in a diet exert different effect on plasma lipid and lipoprotein concentration. SFAs are believed to increase CVD risks by elevating plasma TC and LDL-C, whereas diet rich in MUFAs and PUFAs is able to reduce plasma TC and LDL-C in healthy individuals [37]. The pulp oil of $\mathrm{CO}$ used in this study contained higher MUFAs and PUFAs compared to kernel oil and thus explained the reason of improvement in lipid profile of the NP group. Significant reduction of TG level in rabbits fed on pulp oil (Figure 3(d)) as compared to rabbits fed on kernel oil could be due to the higher unsaturation of the pulp oil. Our result shows that the pulp oil contains more unsaturated fatty acids especially oleic and linoleic acids. The triglyceride-lowering effect of the oil might offer an important approach to prevention of CVD. It is believed that diet containing high unsaturated fatty acids promotes regression of lipid peroxidation process as compared to diet 
high in SFAs [38]. In this study, the supplementation of kernel oil which contains higher SFAs is more prone to lipid peroxidation, indicated by higher plasma MDA level. This could be due to the low antioxidant reserve in the kernel oil indicated by lower antioxidant activity [3] and total phenolic content determined as compared to pulp oil. Moreover, lipid unsaturation is not the only factor influencing the extent of lipid peroxidation. Low antioxidant reserve is another factor influencing higher extent of lipid peroxidation [39].

\subsection{Effect of Pulp and Kernel Oils on Antioxidant Activities} and Plasma Antioxidant Status. Dietary lipids can influence the level of antioxidant enzymes activities. An increase in the activity or expression of these enzymes may protect nucleic acids, amino acid side chains, and the double bonds in unsaturated fatty acids from damage by free radicals [19]. In this study, dietary lipids significantly affected the activity of SOD $(P<0.05)$. Generally, higher SOD activity in both supplemented groups suggesting supplementation of pulp and kernel oils might offer some protective effects against generation of free radicals, thus reducing the oxidative stress by enhancing the conversion of superoxide radicals to hydrogen peroxide $\left(\mathrm{H}_{2} \mathrm{O}_{2}\right)$, followed by deactivation of $\mathrm{H}_{2} \mathrm{O}_{2}$ by GPx [40] (Figures 4(b) and 4(c)).

The higher GPx activity in animal fed on the pulp oil was in agreement with previous study by Leudeu et al. [13] using palm oil that shares similar fatty acid composition. The investigator showed a significant increase $(P<0.05)$ of GPx activity when rats were supplemented with palm oil. Higher GPx activity in NP compared to NK might be related to different amount of phenolic content determined in this study (Table 3). The higher level of phenolic in pulp oil promotes the production of SOD and GPx resulting in the prevention of lipid peroxidation process thus reducing the oxidative stress indicated by lower level of MDA. Plasma total antioxidant status (TAS) measures the antioxidant potential in biological fluids. TAS is defined as the ability of serum or plasma to quench free radical production that consists of a multicompartmental protection against molecular damage of the cell structure [41]. Plasma TAS takes into account the complex interactions that occur between individual antioxidants in vivo [42]. Animals fed on the pulp oil had higher TAS compared to animals fed on the kernel oil. The increase in TAS values is believed to be attributed to the increased activities of GPx and the presence of high phenolic compounds in the pulp oil such as catechin, epigallocatechin gallate and ellagic acid [4], and vitamin E [43]. The interaction of phenolic compounds, vitamin E, and other antioxidant compounds may increase the overall TAS value $[42,44]$.

\section{Conclusions}

Based on findings gathered in this study, it can be concluded that pulp oil appeared to be more protective compared to kernel oil towards CVD biomarkers as it improves the lipid profile with enhancement of the antioxidant status in healthy rabbits thus proving to provide several health benefits.
The decrease in lipid peroxidation level also can reduce the CVD risks. The favorable effects of the pulp oil could be due to the superiority of phenolic, higher MUFAs contents as well as balanced fatty acid content. It can be suggested that pulp oil extracted from CO fruit can be used as healthy vegetable oil in future. Further studies in human subjects, investigation of $\mathrm{CO}$ oil bioactive components as well as its related mechanism in reducing CVD risk are worth studied in future.

\section{Acknowledgments}

The financial support of Science Fund from Ministry of Agriculture, Malaysia (vote no. 5450555) is gratefully acknowledged. The authors also acknowledge the assistance of laboratory staff from the Department of Nutrition and Dietetics, UPM throughout the research project. They also extend their thanks to Universiti Putra Malaysia for the use of laboratory facilities. This research was supported by Universiti Putra Malaysia.

\section{References}

[1] K. Prasad, "Effects of vitamin e on serum enzymes and electrolytes in hypercholesterolemia," Molecular and Cellular Biochemistry, vol. 335, no. 1-2, pp. 67-74, 2010.

[2] A. Azrina, M. N. Nurul Nadiah, and I. Amin, "Antioxidant properties of methanolic extract of Canarium odontophyllum fruit," International Food Research Journal, vol. 17, no. 2, pp. 319-326, 2010.

[3] F. H. Shakirin, K. N. Prasad, A. Ismail, L. C. Yuon, and A. Azlan, "Antioxidant capacity of underutilized Malaysian Canarium odontophyllum (dabai) Miq. fruit," Journal of Food Composition and Analysis, vol. 23, no. 8, pp. 777-781, 2010.

[4] L. Y. Chew, H. E. Khoo, I. Amin, A. Azrina, and C. Y. Lau, "Analysis of phenolic compounds of Dabai (Canarium odontophyllum Miq.) fruits by high-performance liquid chromatography," Food Analytical Methods, pp. 1-12, 2011.

[5] D. Frank, S. Pat, and L. W. Philip, "Improving the analysis of fatty acid methyl esters using retention time locked methods and retention tima database," Agilent Technologies, pp. 1-5, 2006.

[6] T. Gutfinger, "Polyphenols in olive oils," Journal of the American Oil Chemists Society, vol. 58, no. 11, pp. 966-968, 1981.

[7] V. L. Singleton and J. A. Rossi, "Colorimetry of total phenolics with 508 phosphomolybdic-phosphotungstic acid reagent," American Journal of Enology and Viticulture, vol. 16, pp. 144158, 1965.

[8] Y. S. Velioglu, G. Mazza, L. Gao, and B. D. Oomah, "Antioxidant activity and total phenolics in selected fruits, vegetables, and grain products," Journal of Agricultural and Food Chemistry, vol. 46, no. 10, pp. 4113-4117, 1998.

[9] M. Basu, R. Prasad, P. Jayamurthy, K. Pal, C. Arumugam, and R. C. Sawhney, "Anti-atherogenic effects of seabuckhorn (Hipophaea rhamnoides)," Phytomedicine, vol. 14, pp. 770777, 2007.

[10] J. A. Buege and S. D. Aust, "Microsomal lipid peroxidation," Methods in Enzymology, vol. 52, pp. 302-310, 1978.

[11] A. Nurnadia, "The determination of fatty acid composition and vitamin E content in oils extracted from flesh and kernel 
of Canarium odontophyllum," in Department of Nutrition and Dietetics, Universiti Putra Malaysia, Kuala Lumpur, Malaysia, 2007.

[12] H. A. R. Ibrahim, L. B. Lim, S. Salimah, and A. Mariani, "The chemistry of kembayau oil," in Proceedings of the 10th Asean Food Conference, Kuala Lumpur, Malaysia, August 2007.

[13] B. C. T. Leudeu, C. Tchiegang, M. D. Gadet et al., "Effect of Canarium SCheweinfurthii and Dacrydodes edulis oils on blood lipids, lipid peroxidation and oxidative stress in rats," Journal of Food Technology, vol. 41, pp. 385-390, 2006.

[14] J. Milosevic, D. S. Ashton, and M. J. Cocksedge, "Lipids present in olive skin," International Journal of Food Science and Technology, vol. 37, no. 5, pp. 523-526, 2002.

[15] F. Takenaga, K. Matsuyama, S. Abe, Y. Torii, and S. Itoh, "Lipid and fatty acid composition of mesocarp and seed of avocado fruits harvested at northern range in Japan," Journal of Oleo Science, vol. 57, no. 11, pp. 591-597, 2008.

[16] B. Fang, L. Jiang, and F. Z. Ren, "Effects of initial heating temperature on the crystallization rate of trans-free palm oil," Journal of Thermal Analysis and Calorimetry, vol. 100, no. 3, pp. 1085-1090, 2010.

[17] Y. Kakuda, F. Jahaniaval, M. F. Marcone, L. Montevirgen, Q. Montevirgen, and J. Umali, "Characterization of pili nut (Canarium ovatum) oil: fatty acid and triacylglycerol composition and physicochemical properties," Journal of the American Oil Chemists' Society, vol. 77, no. 9, pp. 991-997, 2000.

[18] Z. He and W. Xia, "Nutritional composition of the kernels from Canarium album L.," Food Chemistry, vol. 102, no. 3, pp. 808-811, 2007.

[19] R. Albertini, R. Moratti, and G. De Luca, "Oxidation of lowdensity lipoprotein in atherosclerosis from basic biochemistry to clinical studies," Current Molecular Medicine, vol. 2, no. 6, pp. 579-592, 2002.

[20] N. Pellegrini, F. Visioli, S. Buratti, and F. Brighenti, "Direct analysis of total antioxidant activity of olive oil and studies on the influence of heating," Journal of Agricultural and Food Chemistry, vol. 49, no. 5, pp. 2532-2538, 2001.

[21] G. Beltrán, M. P. Aguilera, C. D. Rio, S. Sanchez, and L. Martinez, "Influence of fruit ripening process on the natural antioxidant content of Hojiblanca virgin olive oils," Food Chemistry, vol. 89, no. 2, pp. 207-215, 2005.

[22] B. Dimitrios, "Sources of natural phenolic antioxidants," Trends in Food Science and Technology, vol. 17, no. 9, pp. 505512, 2006.

[23] C. I. G. Tuberoso, A. Kowalczyk, E. Sarritzu, and P. Cabras, "Determination of antioxidant compounds and antioxidant activity in commercial oilseeds for food use," Food Chemistry, vol. 103, no. 4, pp. 1494-1501, 2007.

[24] B. J. Rolls and D. J. Shide, "The influence of dietary fat on food intake and body weight," Nutrition Reviews, vol. 50, no. 10, pp. 283-290, 1992.

[25] A. Astrup, L. Ryan, G. K. Grunwald et al., "The role of dietary fat in body fatness: evidence from a preliminary meta-analysis of ad libitum low-fat dietary intervention studies," British Journal of Nutrition, vol. 83, supplement 1, pp. S25-S32, 2000.

[26] B. S. Tzang, S. F. Yang, S. G. Fu, H. C. Yang, H. L. Sun, and Y. C. Chen, "Effects of dietary flaxseed oil on cholesterol metabolism of hamsters," Food Chemistry, vol. 114, no. 4, pp. 1450-1455, 2009.

[27] M. B. Katan, P. L. Zock, and R. P. Mensink, "Effects of fats and fatty acids on blood lipids in humans: an overview," American Journal of Clinical Nutrition, vol. 60, supplement 6, pp. 1017S1022S, 1994.
[28] F. B. Hu, J. E. Manson, and W. C. Willett, "Types of dietary fat and risk of coronary heart disease: a critical review," Journal of the American College of Nutrition, vol. 20, no. 1, pp. 5-19, 2001.

[29] T. A. Wilson, R. J. Nicolosi, T. Kotyla, K. Sundram, and D. Kritchevsky, "Different palm oil preparations reduce plasma cholesterol concentrations and aortic cholesterol accumulation compared to coconut oil in hypercholesterolemic hamsters," Journal of Nutritional Biochemistry, vol. 16, no. 10, pp. 633-640, 2005.

[30] K. G. Nevin and T. Rajamohan, "Beneficial effects of virgin coconut oil on lipid parameters and in vitro LDL oxidation," Clinical Biochemistry, vol. 37, no. 9, pp. 830-835, 2004.

[31] O. B. Ajayi and D. D. Ajayi, "Effect of oilseed diets on plasma lipid profile in albino rats," Pakistan Journal of Nutrition, vol. 8, no. 2, pp. 116-118, 2009.

[32] M. Gonzalez-Santiago, E. Martin-Baustista, J. J. Carrero et al., "One-month administration of hydrotyrosol, a phenolic antioxidant present in olive oil, to hyperlipidemic rabbits improves blood lipid profile, antioxidant statusand reduces atherosclerotic development," Atherosclerosis, vol. 188, pp. 3542, 2006.

[33] K. Ogasawara, S. Mashiba, H. Hashimoto et al., "Low-density lipoprotein (LDL) which includes apolipoprotein A-I (apoAILDL) as a novel maker of coronary artery disease," Clinical Chimica, vol. 397, pp. 42-47, 2008.

[34] C. C. Chen, L. K. Liu, J. D. Hsu, H. P. Huang, M. Y. Yang, and C. J. Wang, "Mulberry extract inhibits the development of atherosclerosis in cholesterol-fed rabbits," Food Chemistry, vol. 91, no. 4, pp. 601-607, 2005.

[35] P. Matilla, J. Hellstrom, and R. Torronen, "Phenolic acids in berries, fruits and beverages," Journal of Agricultural Food Chemistry, vol. 19, pp. 7193-7199, 2006.

[36] M. D. Salvador, F. Aranda, and G. Fregapane, "Influence of fruit ripening on 'Cornicabra' virgin olive oil quality: a study of four successive crop seasons," Food Chemistry, vol. 73, no. 1, pp. 45-53, 2001.

[37] T. A. B. Sanders, "Fat and fatty acid intake and metabolic effects in the human body," Annals of Nutrition and Metabolism, vol. 55, no. 1-3, pp. 162-172, 2009.

[38] Y. F. Lu and S. Lu, "Influence of dietary fat saturation on lipid peroxidation of serum and low density lipoprotein in rats," Nutrition Research, vol. 22, no. 4, pp. 463-472, 2002.

[39] E. R. Miller, L. J. Appel, and T. H. Risby, "Effect of dietary patterns on measures of lipid peroxidation: results from a randomized clinical trial," Circulation, vol. 98, no. 22, pp. 2390-2395, 1998.

[40] D. D. Heistad, Y. Wakisaka, J. Miller, Y. Chu, and R. Pena-Silva, "Novel aspects of oxidative stress in cardiovascular diseases," Circulation Journal, vol. 73, no. 2, pp. 201-207, 2009.

[41] H. Sies, "Total antioxidant capacity: appraisal of a concept," Journal of Nutrition, vol. 137, no. 6, pp. 1493-1495, 2007.

[42] V. Fenkci, S. Fenkci, M. Yilmazer, and M. Serteser, "Decreased total antioxidant status and increased oxidative stress in women with polycystic ovary syndrome may contribute to the risk of cardiovascular disease," Fertility and Sterility, vol. 80, no. 1, pp. 123-127, 2003.

[43] A. Azrina, I. Amin, and I. Muhammad, "Proximate composition and characterization of fat in Canarium odontophyllum," in Proceedings of the 10th Asean Food Conference, 2007.

[44] A. Ghiselli, M. Serafini, F. Natella, and C. Scaccini, "Total antioxidant capacity as a tool to assess redox status: critical view and experimental data," Free Radical Biology and Medicine, vol. 29, no. 11, pp. 1106-1114, 2000. 


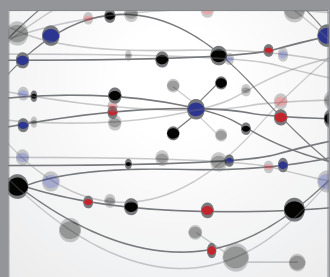

The Scientific World Journal
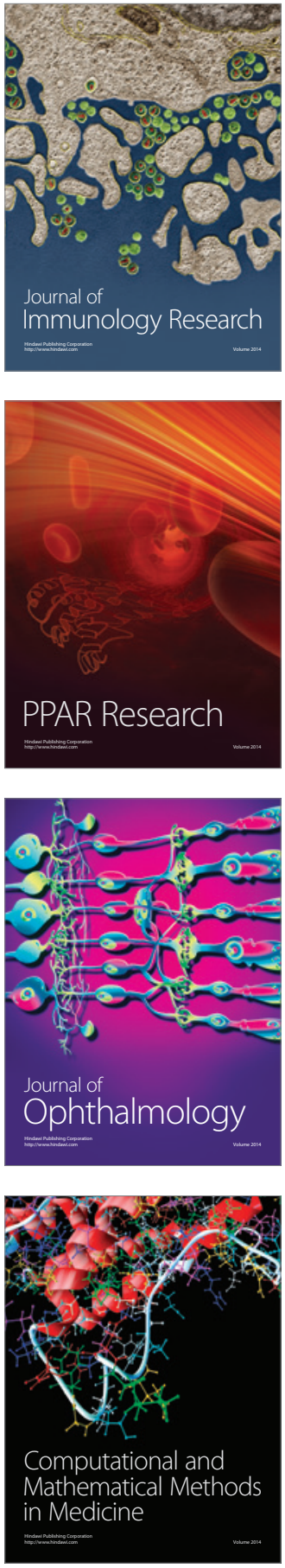

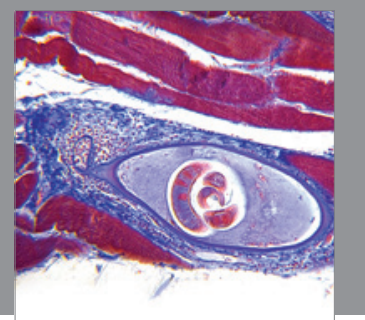

Gastroenterology

Research and Practice
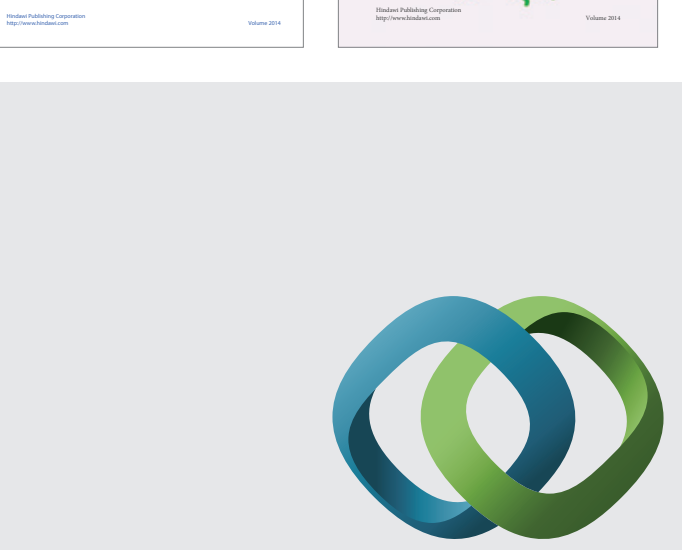

\section{Hindawi}

Submit your manuscripts at

http://www.hindawi.com
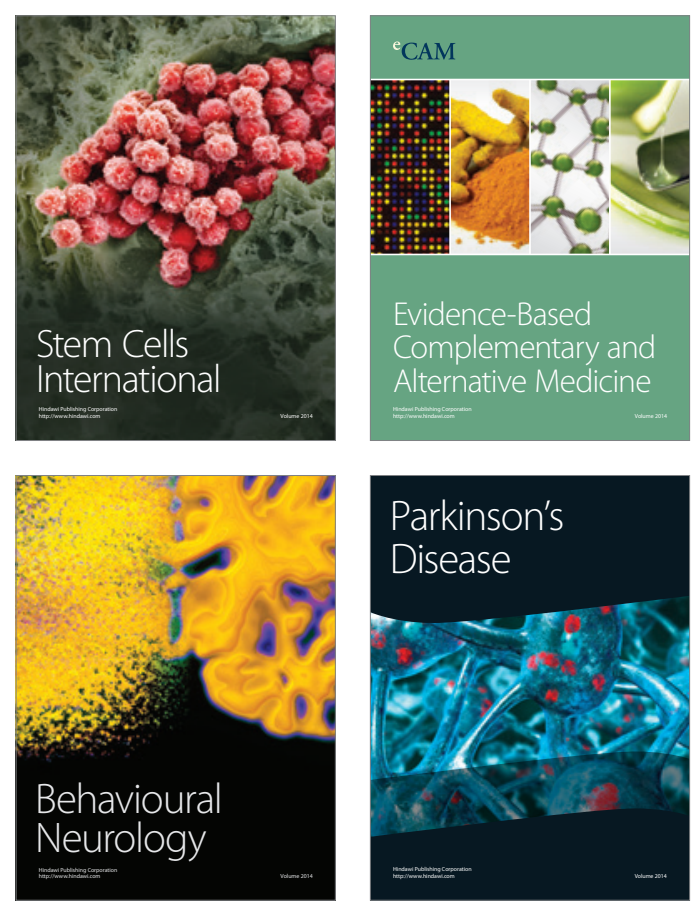

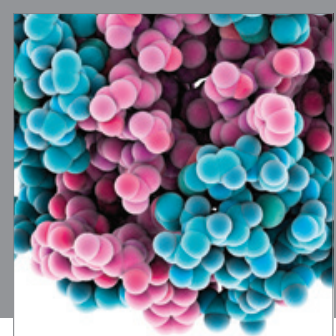

Journal of
Diabetes Research

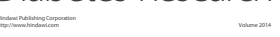

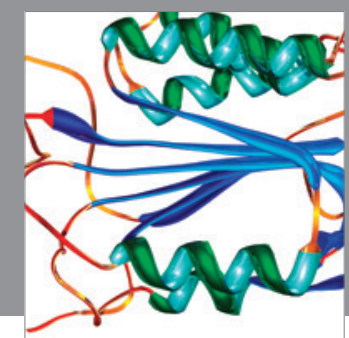

Disease Markers
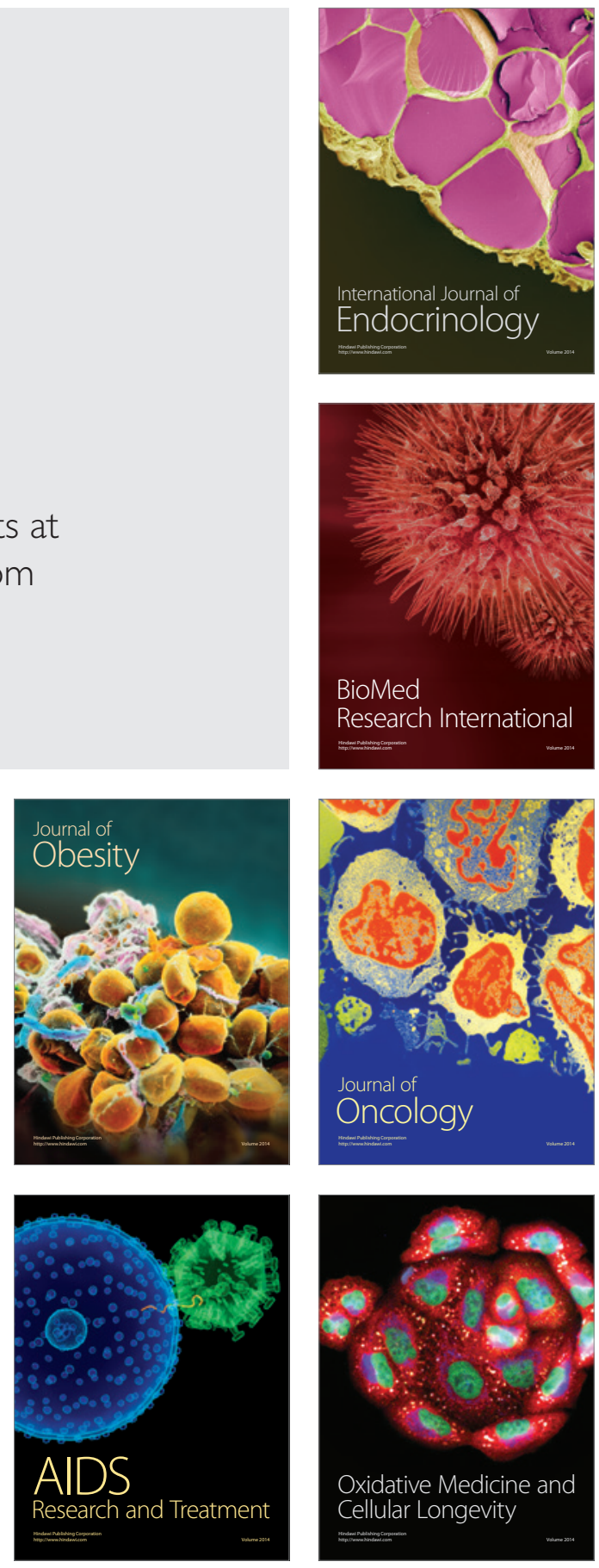